\title{
Revisiting the Correspondence between Cut Elimination and Normalisation
}

\author{
José Espírito Santo* \\ Laboratory for Foundations of Computer Science \\ Division of Informatics, The University of Edinburgh, James Clerk Maxwell Building, \\ The King's Buildings, Mayfield Road, Edinburgh EH9 3JZ, Scotland UK \\ jes@dcs.ed.ac.uk
}

\begin{abstract}
Cut-free proofs in Herbelin's sequent calculus are in 1-1 correspondence with normal natural deduction proofs. For this reason Herbelin's sequent calculus has been considered a privileged middle-point between L-systems and natural deduction. However, this bijection does not extend to proofs containing cuts and Herbelin observed that his cutelimination procedure is not isomorphic to $\beta$-reduction.

In this paper we equip Herbelin's system with rewrite rules which, at the same time: (1) complete in a sense the cut elimination procedure firstly proposed by Herbelin; and (2) perform the intuitionistic "fragment" of the tq-protocol - a cut-elimination procedure for classical logic defined by Danos, Joinet and Schellinx. Moreover we identify the subcalculus of our system which is isomorphic to natural deduction, the isomorphism being with respect not only to proofs but also to normalisation.

Our results show, for the implicational fragment of intuitionistic logic, how to embed natural deduction in the much wider world of sequent calculus and what a particular cut-elimination procedure normalisation is.
\end{abstract}

\section{Introduction}

In his paper about a " $\lambda$-calculus structure" isomorphic to a "Gentzen-style sequent calculus structure" [6], Herbelin proposed to define a $\lambda$-like calculus corresponding to a $L J$-like sequent calculus in the same way as $\lambda$-calculus corresponds to natural deduction.

Herbelin starts by refining the simplest, many-one assignment of terms to sequent calculus proofs, usually denoted by $\varphi$ and that comes from the theory of the relationship between sequent calculus and natural deduction $[10,15,9$, $11,3]$. The refinement is to consider a restriction of $L J$ called $L J T$ (respec. a term calculus called $\bar{\lambda}$-calculus) whose cut-free proofs (respec. cut-free terms) are in 1-1 correspondence with normal natural deduction proofs (respec. normal $\lambda$-terms).

\footnotetext{
* The author is supported by Fundação para a Ciência e Tecnologia, Portugal.
} 
Dyckhoff and Pinto $[2,3]$ showed the merits of the cut-free fragment of $L J T$ as a proof-theoretical tool and emphasized its privileged intermediate position between sequent calculus and natural deduction. The purpose of this paper is to define an intermediate system of this kind for proofs possibly containing cuts and a cut-elimination procedure which together give an isomorphic copy of natural deduction in sequent calculus format, with respect not only to proofs but also to proof normalisation.

Full $L J T$ is not the solution to this problem. The bijection with natural deduction does not extend to proofs with cuts and Herbelin observed that his cut-elimination procedure fails to implement full $\beta$-reduction (it just implements a strategy).

The $\bar{\lambda}$-calculus includes an operator of explicit substitution so that local steps of cut permutation can be written as elementary steps of substitution propagation (calculi of explicit substitution for similar purposes can be found in $[14,5,13])$. Instead of making substitution explicit, we perform the complete upwards permutation of a cut in a single step of reduction by a global operation. This is inspired in the so-called tq-protocol, a cut-elimination procedure for classical "coloured" proofs defined by Danos, Joinet and Schellinx [1].

We equip $L J T$ with a reduction procedure of this kind which completes, in a sense, $L J T$ 's original procedure, obtaining a sequent calculus and corresponding $\lambda$-calculus which we call $H J^{+}$and $\lambda_{H}^{+}$, respectively. We prove that $H J^{+}$is just performing the intuitionistic "fragment" of the tq-protocol and that the typable subcalculus of $\lambda_{H}^{+}$is strongly normalising and confluent. Furthermore, we identify natural subsystems $H J$ and $\lambda_{H}$ such that $H J$ (respec. $\lambda_{H}$ ) is isomorphic, in the strong sense required above, to $N J$ (respec. $\lambda$ ). In particular, both $\lambda_{H}^{+}$and $\lambda_{H}$ implement full $\beta$-reduction.

The reader finds in Table 1 (where inc stands for inclusion) a map of systems and translations which will appear in the following.

\section{Notations and Terminology}

We just treat intuitionistic implicational logic (implication written as $\supset$ ). Barendregt's convention applies to all calculi in this paper. A context is a consistent set of declarations $x: A$. By consistent we mean that if $x: A$ and $x: B$ are in a context, then $A=B$. Contexts are ranged over by $\Gamma$. We write $x \in \Gamma$ meaning $x: A \in \Gamma$ for some $A . \Gamma, x: A$ denotes the consistent union $\Gamma \cup\{x: A\}$, which means that, if $x$ is already declared in $\Gamma$, then it is declared with type $A$.

We call left (respec. right) subderivation of a cut instance the subderivation in which the cutformula occurs in the RHS (respec. LHS) of its endsequent. Such cutformula is called the left (respec. right) cutformula of that instance.

\section{Background}

\subsection{Herbelin's $L J T$ and $\bar{\lambda}$-calculus}

We refer to [6] for details about $L J T$ and $\bar{\lambda}$. We adopt some simplification of syntax introduced in [2] (but the numbering of cuts is different!). Table 2 presents 
Table 1. Map of systems and translations

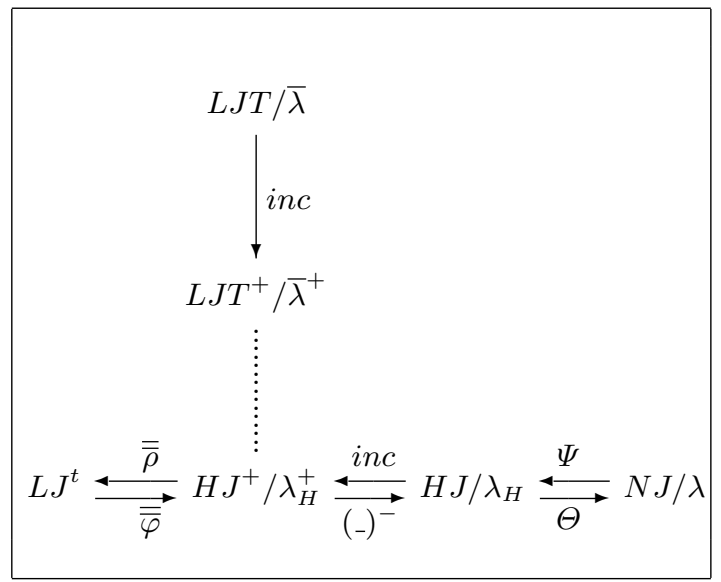

the syntax and typing rules of $\bar{\lambda}$ (and thus the inference rules of $L J T$ ) and the reduction rules of $\bar{\lambda}$ which define the cut-elimination procedure of $L J T$ (Der stands for Dereliction).

In $\bar{\lambda}$ there are two kinds of expressions: terms and lists of terms. The term $x\left[t_{1}, \ldots, t_{n}\right]$ can be seen as the $\lambda$-term $\left(\ldots\left(x t_{1}\right) \ldots t_{n}\right)$ but with the advantage of having the head-variable at the surface. $t\{x:=v\}$ and $l\{x:=v\}$ are explicit substitution operators and $l l^{\prime}$ is an explicit append of list (cf. the reduction rules). Notice that in $t\{x:=v\}$ and $l\{x:=v\}, x$ is bound and $x \notin F V(v)$.

There are two kinds of derivable sequents: $\Gamma ;-\vdash t: B$ and $\Gamma ; A \vdash l: B$. In both there is a distinguished position in the LHS called stoup. The crucial restriction of $L J T$ is that the rule $L \supset$ introduces $A \supset B$ in the stoup and $B$ has to be in the stoup of the right subderivation's endsequent. Forget for a second rules cut $_{2}$ and cut $_{4}$. In this case (in particular in cut-free $L J T$ ), besides $A x$, no rule can introduce a formula in the stoup and thus the last rule of the right subderivation of an instance of $L \supset$ is again $L \supset$ and so on until $A x$ is reached.

There are two kinds of cuts (head-cut and mid-cut) according to whether the right cutformula is in the stoup or not. Notice that in the reduction rules there are no permutation of cuts.

\section{$2.2 L^{t}$ and the Intuitionistic "tq-protocol"}

Table 3 presents the sequent calculus $L J^{t}$ and a corresponding, nameless term calculus in which a cut-elimination procedure is expressed.

We leave to the reader to provide the definitions of free and bound variable in a term $L$. The idea is that, in $\mathrm{L}\left(x, L_{1},(y) L_{2}\right), x$ occurs free and $y$ bound. By Barendregt's convention, neither $y$ occurs free in $L_{1}$ nor $x$ occurs bound in $L_{1}$ or 
Table 2. Herbelin's LJT and $\bar{\lambda}$-calculus

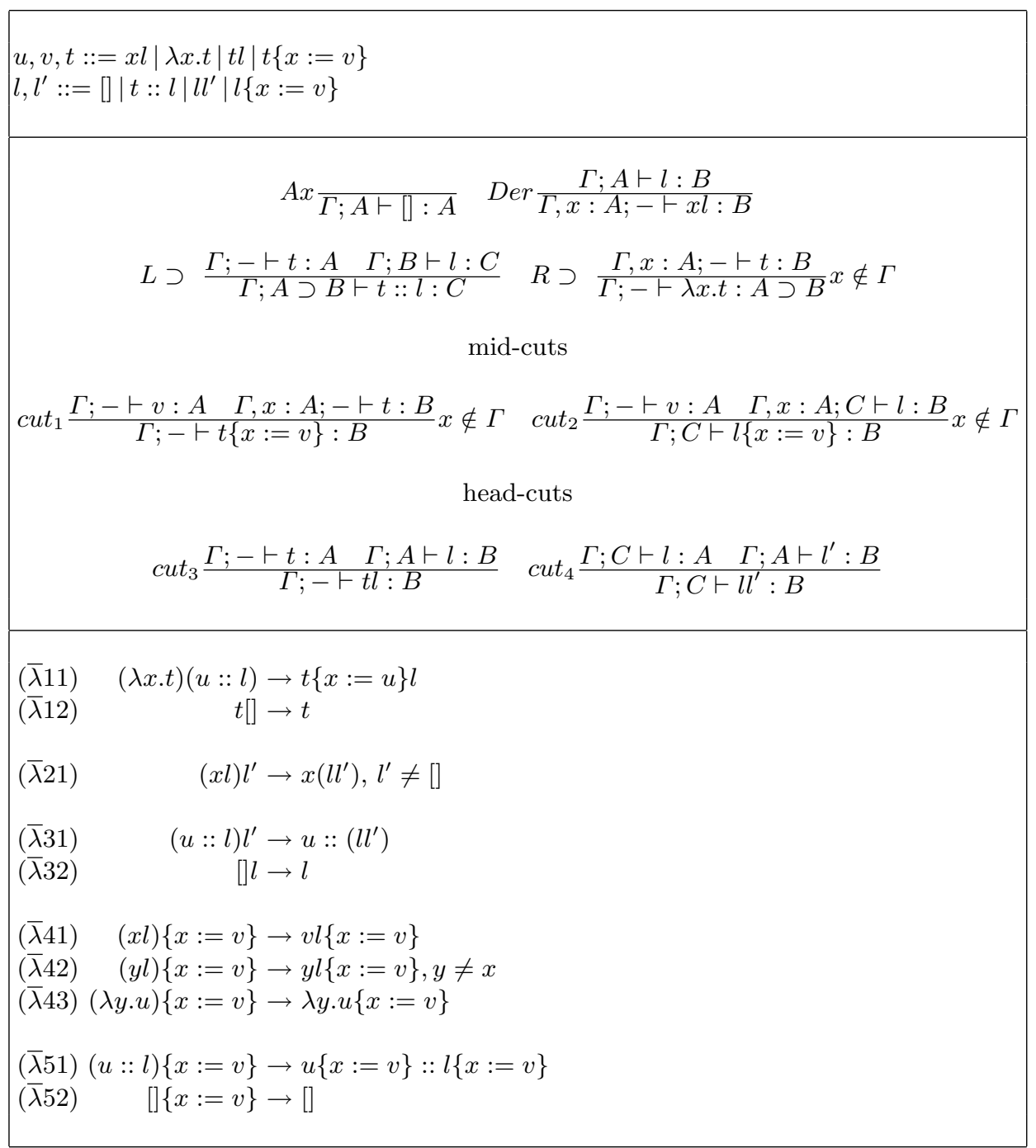


Table 3. $L J^{t}$

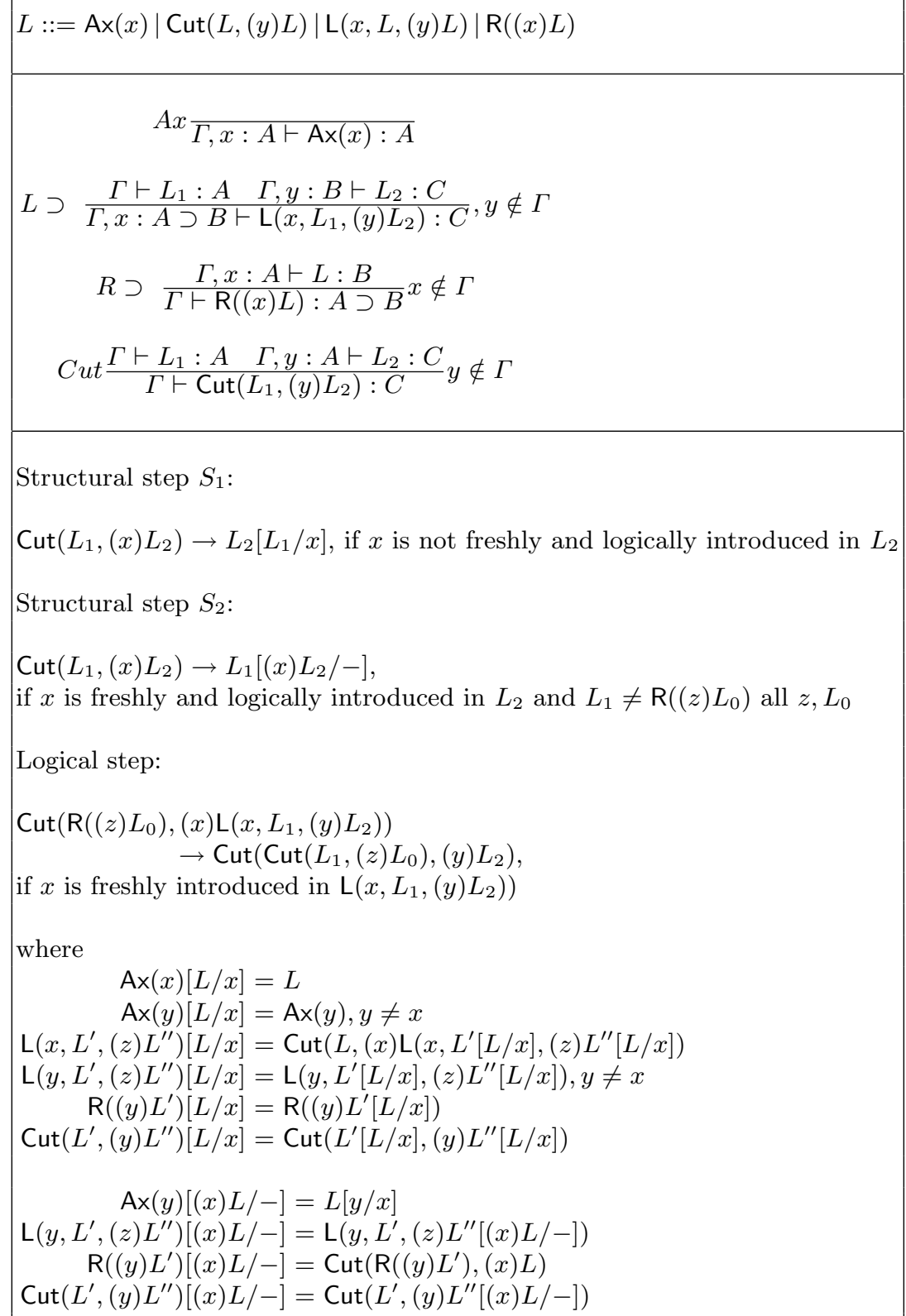


$L_{2}$, although $x$ may occur free in $L_{1}$ or $L_{2}$ (meaning that an implicit contraction is happening).

The cut-elimination procedure is a "fragment" of the so-called tq-protocol, a strongly normalising and confluent procedure for classical, "coloured" proofs defined in [1]. To be precise, it is the restriction of the tq-protocol to intuitionistic, t-coloured proofs in which an "orientation" of the multiplicative connective $\supset$ has been fixed.

Roughly, the protocol works as follows: a cut is firstly permuted upwards through its right subderivation (structural step $S_{1}$ ) and then through its left subderivation (structural step $S_{2}$ ) until it becomes a logical cut, to which the logical step applies, giving rise to new cuts of lower degree. A logical cut is a cut whose both cutformulas are freshly and logically introduced, i.e. introduced by a logical rule $(L \supset$ or $R \supset$ ) without implicit contraction. An equivalent description of step $S_{1}$ (respec. step $S_{2}$ ) is: to push the left (respec. right) subderivation upwards through the "tree of ancestors" [1] of the right (respec. left) cutformula.

The operations $L_{2}\left[L_{1} / x\right]$ and $L_{1}\left[(x) L_{2} /-\right]$ implement the structural steps $S_{1}$ and $S_{2}$, respectively, and are inspired in the operations of substitution and co-substitution defined by Urban and Bierman in [12].

\section{$3 \quad \mathrm{HJ}^{+}$and the $\lambda_{H^{+}}^{+}$calculus}

We refer to Table 4 for the definition of $H J^{+}$and $\lambda_{H}^{+}$. The motivation for these systems rests in the following observations.

The "life cycle" of a cut in $L J T$ has three stages. It starts as a mid-cut and the first stage is a upwards permutation through its right subderivation, performed by rules $\bar{\lambda} 4 i$ and $\bar{\lambda} 5 j$. The goal is to generate head-cuts (see rule $\bar{\lambda} 41)$. The operation subst performs this permutation in a single step. In doing so, cuts of the form $l\{x:=v\}$ become "internal" to this process and hence are not needed in the syntax. Now observe that in $L J T$ such permutation of a midcut can complete only if, in its course, we do not need to permute this mid-cut with another cut. This is why, in the definition of subst, extra clauses occur corresponding to the permutations

$$
\begin{aligned}
(\bar{\lambda} 44) \quad(t l)\{x:=v\} & \rightarrow t\{x:=v\} l\{x:=v\}, \\
(\bar{\lambda} 45) t\{y:=u\}\{x:=v\} & \rightarrow t\{x:=v\}\{y:=u\{x:=v\}\} .
\end{aligned}
$$

Let us return to the head-cuts generated by the first stage. Notice that in a head-cut $v l$, if $l \neq[]$ then its right cutformula is freshly and logically introduced. Such a cut is permuted upwards through its left subderivation by the rules $\bar{\lambda} 21$ and $\bar{\lambda} 3 i$, generating along the way $\bar{\lambda} 11$-redexes, i.e. logical cuts in the $L J^{t}$ sense. The last stage of the "life cycle" of these logical cuts is $\bar{\lambda} 11$-reduction, by which cuts of lower degree are generated.

Again the operation insert performs in a single step the permutations of the second stage and cuts $l l^{\prime}$ become "internal" and thus superfluous in the syntax. Extra clauses in insert's definition correspond to the permutations 
Table 4. $H J^{+}$and $\lambda_{H}^{+}$-calculus

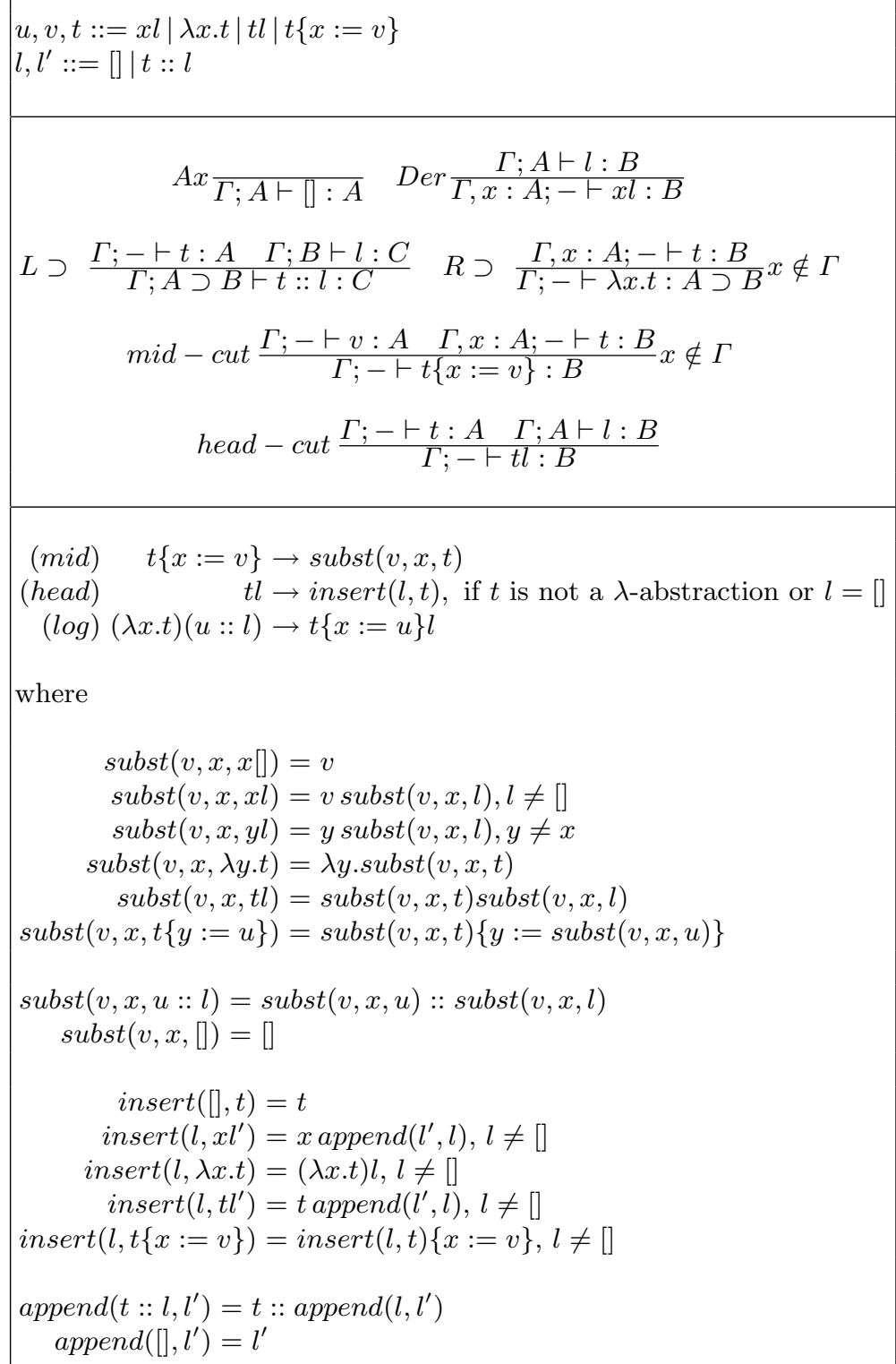




$$
\begin{aligned}
& (\bar{\lambda} 22) \quad\left(t l^{\prime}\right) l \rightarrow t\left(l^{\prime} l\right), \\
& (\bar{\lambda} 23)(t\{x:=v\}) l \rightarrow(t l)\{x:=v\} .
\end{aligned}
$$

Define $L J T^{+}$as $L J T$ plus the four new reduction rules just presented. We leave to the reader the formalisation of the obvious relations between $L J T$, $L J T^{+}$and $H^{+}$.

On the other hand, it should be clear that reductions $\rightarrow_{m(i d)}$ and $\rightarrow_{h(\text { ead })}$ in $H J^{+}$have a strong connection with the structural steps $S_{1}$ and $S_{2}$, respectively, of $L J^{t}$ and that, roughly (but not exactly), a mid-cut is a $S_{1}$-redex and a headcut is a $S_{2}$-redex. This is formalised by defining a map $\overline{\bar{\rho}}: H J^{+} \rightarrow L J^{t}$ as in Table 5 (where $z \notin F V(l)$ in the second and last clauses of the definition of $\overline{\bar{\rho}}$ ).

Table 5. Translations $\overline{\bar{\rho}}$ and $\overline{\bar{\varphi}}$

\begin{tabular}{|r|r|}
\hline$\overline{\bar{\rho}}(x[])$ & $=\mathrm{Ax}(x)$ \\
$\overline{\bar{\rho}}(x(t:: l))=\mathrm{L}(x, \overline{\bar{\rho}}(t),(z) \overline{\bar{\rho}}(z l))$ & $\overline{\bar{\varphi}}(\mathrm{Ax}(x))=x[]$ \\
$\overline{\bar{\rho}}(\lambda x \cdot t)=\mathrm{R}((x) \overline{\bar{\rho}}(t))$ & $\overline{\bar{\varphi}}(\mathrm{R}((x) L))=\lambda x \cdot \overline{\bar{\varphi}}(L)$ \\
$\overline{\bar{\rho}}(t\{x:=v\})=\operatorname{Cut}(\overline{\bar{\rho}}(v),(x) \overline{\bar{\rho}}(t))$ & $\overline{\bar{\varphi}}\left(\mathrm{L}\left(x, L_{1},(y) L_{2}\right)\right)=\overline{\bar{\varphi}}\left(L_{2}\right)\left\{y:=x\left[\overline{\bar{\varphi}}\left(L_{1}\right)\right]\right\}$ \\
$\overline{\bar{\rho}}(t l)=\operatorname{Cut}(\overline{\bar{\rho}}(t),(z) \overline{\bar{\rho}}(z l))$ & $\overline{\bar{\varphi}}\left(\operatorname{Cut}\left(L_{1},(y) L_{2}\right)\right)=\overline{\bar{\varphi}}\left(L_{2}\right)\left\{y:=\overline{\bar{\varphi}}\left(L_{1}\right)\right\}$ \\
&
\end{tabular}

Lemma 1. $\overline{\bar{\rho}}(\operatorname{subst}(v, x, t))=\overline{\bar{\rho}}(t)[\overline{\bar{\rho}}(v) / x]$, if $x \notin F V(v)$.

Proposition 1. If $t \rightarrow_{m} t^{\prime}$ in $H J^{+}$then either $\overline{\bar{\rho}}(t) \rightarrow_{S_{1}} \overline{\bar{\rho}}\left(t^{\prime}\right)$ or $\overline{\bar{\rho}}(t)=\overline{\bar{\rho}}\left(t^{\prime}\right)$ in $L J^{t}$.

The single anomaly is a mid-step of the form

$$
(x l)\{x:=v\} \rightarrow_{m} v l,
$$

where $x \notin F V(l)$, which collapses in $L J^{t}$ because $\overline{\bar{\rho}}((x l)\{x:=v\})=\overline{\bar{\rho}}(v l)$. There is another (and last) anomaly, this time regarding head-cuts. The term $t[]$ is a $S_{1}$-redex and not a $S_{2}$-redex. We can split $\rightarrow_{h}$ as

$\left(h_{1}\right) t[] \rightarrow t$

$\left(h_{2}\right) \quad t l \rightarrow \operatorname{insert}(l, t)$, if $t$ is not a $\lambda$-abstraction and $l \neq[]$

and then:

Lemma 2. $\overline{\bar{\rho}}($ insert $(l, t))=\overline{\bar{\rho}}(t)[(z) \overline{\bar{\rho}}(z l) /-]$, if $z \notin F V(l)$ and $l \neq[]$.

Proposition 2. If $t \rightarrow_{h_{i}} t^{\prime}$ in $H J^{+}$then $\overline{\bar{\rho}}(t) \rightarrow_{S_{i}} \overline{\bar{\rho}}\left(t^{\prime}\right)$ in $L J^{t}, i=1,2$. 
Finally:

Proposition 3. If $t \rightarrow_{\log } t^{\prime}$ in $H J^{+}$then $\overline{\bar{\rho}}(t) \rightarrow_{\text {Log }} \overline{\bar{\rho}}\left(t^{\prime}\right)$ in $L J^{t}$.

Corollary 1. The typable terms of $\lambda_{H}^{+}$are strongly normalising.

Proof. By strong normalisation of the tq-protocol, Propositions 1,2,3 and the fact that there can be no infinite reduction sequence starting from a $\lambda_{H}^{+}$-term and only made of steps (1).

In the following it will be useful, namely for proving confluence of $\lambda_{H}^{+}$, to consider a translation $\overline{\bar{\varphi}}: L J^{t} \rightarrow H J^{+}$, as defined in Table 5 .

\section{$4 \quad \boldsymbol{H} \boldsymbol{J}$ and the $\boldsymbol{\lambda}_{\boldsymbol{H}}$-calculus}

$H J$ (see Table 6 ) was obtained by simplifying $H J^{+}$in such a way that the new calculus could be proved isomorphic to $N J$ by means of functions $\Psi, \Theta$ extending those defined in [2] between cut-free $L J T$ and normal $N J$.

The first thing to do is to get rid of mid-cuts and $\rightarrow_{m}$. This requires that $l o g$ becomes

$$
(\lambda x . t)(u:: l) \rightarrow \operatorname{subst}(u, x, t) l .
$$

However this is not enough. One problem is that we would have $\Theta(t[])=\Theta(t)$ and thus $\Theta$ would not be injective. Hence we must require $l \neq[]$ in every headcut $t l$. The second problem is that $\Psi(M)$ will be $h$-normal, for all $\lambda$-term $M$. This requires two measures: (a) We restrict ourselves to $h$-normal terms. When mid-cuts are dropped, $t(u:: l)$ is $h$-normal iff $t$ is a $\lambda$-abstraction. Thus headcuts are required to be of the restricted form $(\lambda x . t)(u:: l)$. (b) We drop $\rightarrow_{h}$ and have to reduce immediately, by performing insert, the $h$-redexes generated in (2). Now $\operatorname{subst}(u, x, t) l$ can itself be a $h$-redex and the $h$-redex $u l^{\prime}$ may be created at subformulas of $t$ of the form $x l^{\prime}$. This explains the first clause in the new definition of subst in $H J$ and the new version of (2) which we call $\beta_{H}$.

Every $\lambda_{H}$-term is a $\lambda_{H}^{+}$-term and next proposition says that $\beta_{H}$ is a packet of several steps of reduction in $\mathrm{HJ}^{+}$and, indirectly, in the tq-protocol.

Proposition 4. If $t \rightarrow_{\beta_{H}} t^{\prime}$ in $H J$ then $t \rightarrow_{l, m, h}^{+} t^{\prime}$ in $H J^{+}$.

Conversely, there is a translation $\left({ }_{-}^{-}\right)^{-}: H J^{+} \rightarrow H J$ defined by:

$$
\begin{aligned}
(x l)^{-} & =x l^{-}, \\
(\lambda x . t)^{-} & =\lambda x \cdot t^{-} \\
(t l)^{-} & =\operatorname{insert}\left(l^{-}, t^{-}\right), \\
(t\{x:=v\})^{-} & =\operatorname{subst}\left(v^{-}, x, t^{-}\right), \\
(u:: l)^{-} & =u^{-}:: l^{-}, \\
([])^{-} & =[] .
\end{aligned}
$$

Define $\bar{\rho}$ as the restriction of $\overline{\bar{\rho}}$ to $H J$ and $\bar{\varphi}=\left({ }_{-}\right)^{-} \circ \overline{\bar{\varphi}}$. These $\bar{\rho}, \bar{\varphi}$ extend those of [3]. 
Table 6. $H J$ and $\lambda_{H \text {-calculus }}$

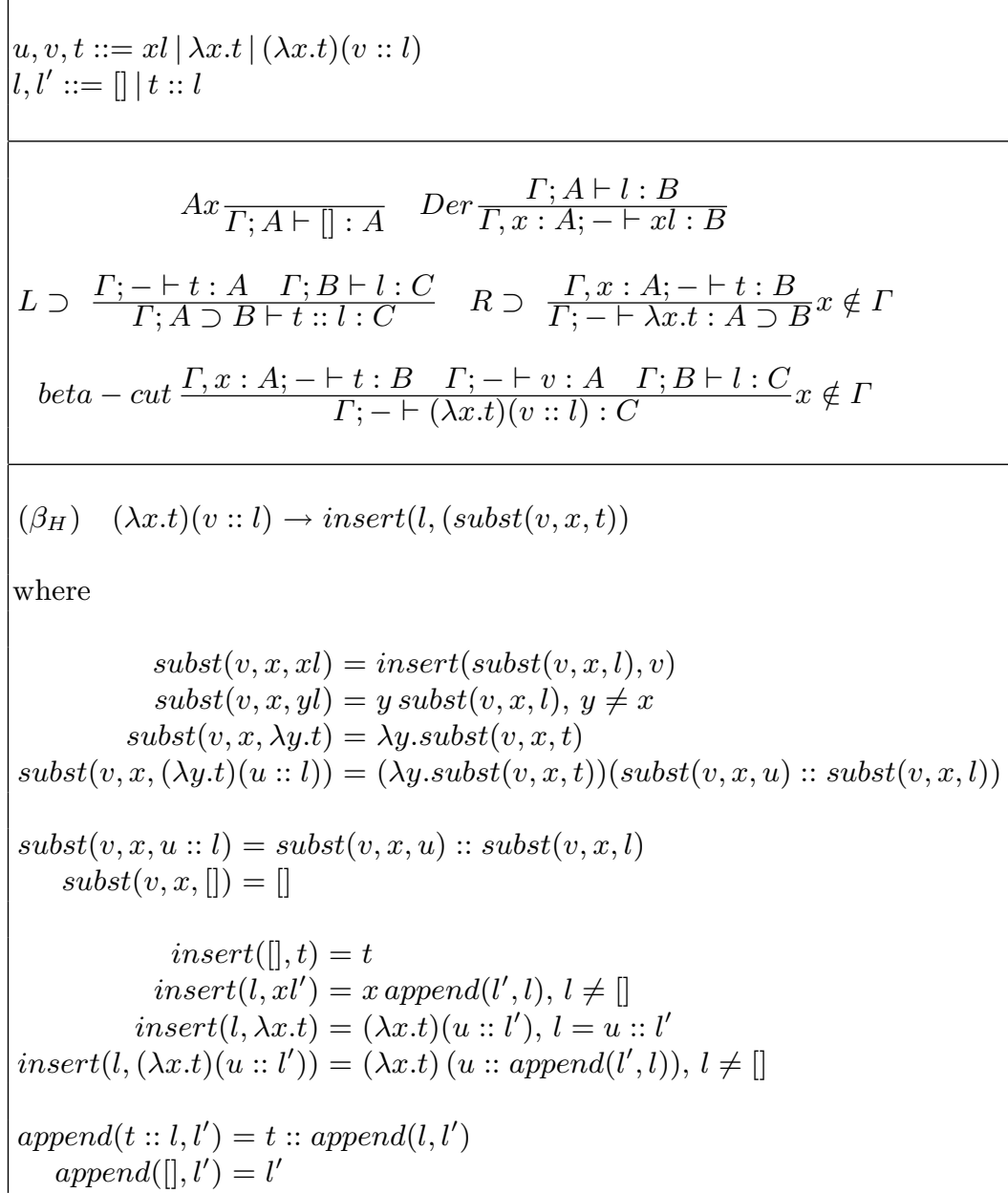


Proposition 5. $\bar{\varphi} \circ \bar{\rho}=i d$.

Corollary 2. The typable subcalculs of $\lambda_{H}^{+}$is confluent.

Proof. Since the typable subcalculus of $\lambda_{H}^{+}$is strongly normalising, it suffices, by Newman's Lemma, to prove uniqueness of normal forms. Suppose $t \rightarrow^{*} t_{1}, t_{2}$ and that both $t_{i}$ are cut-free. By the simulation results above, $\overline{\bar{\rho}}(t) \rightarrow^{*} \overline{\bar{\rho}}\left(t_{1}\right), \overline{\bar{\rho}}\left(t_{2}\right)$ and, since $\overline{\bar{\rho}}$ preserves cut-freeness, both $\overline{\bar{\rho}}\left(t_{i}\right)$ are cut-free. As $\overline{\bar{\rho}}$ preserves typability, $\overline{\bar{\rho}}\left(t_{1}\right)$ and $\overline{\bar{\rho}}\left(t_{2}\right)$ are obtained within the tq-protocol and, by confluence of the tq-protocol, $\overline{\bar{\rho}}\left(t_{1}\right)=\overline{\bar{\rho}}\left(t_{2}\right)$. Now crucially $t_{1}, t_{2} \in H J$ because $t_{1}, t_{2}$ are cut-free. Then, $t_{1}=\bar{\varphi}\left(\bar{\rho}\left(t_{1}\right)\right)=\bar{\varphi}\left(\overline{\bar{\rho}}\left(t_{1}\right)\right)=\bar{\varphi}\left(\overline{\bar{\rho}}\left(t_{2}\right)\right)=\bar{\varphi}\left(\bar{\rho}\left(t_{2}\right)\right)=t_{2}$.

Now let us turn to the relation between $H J$ and $N J$. It is convenient to give the syntax of $\lambda$-calculus as

$$
\begin{aligned}
M, N & ::=x|\lambda x \cdot M| \operatorname{app}(A) \\
A & ::=x M|(\lambda x \cdot M) N| A M .
\end{aligned}
$$

Translations $\Psi$ and $\Theta$ between $H J$ and $N J$ are given in Table 7 .

\begin{tabular}{|c|c|}
\hline $\begin{aligned} \Psi(x) & =x[] \\
\Psi(\lambda x . M) & =\lambda x \cdot \Psi M \\
\Psi(\operatorname{app}(A)) & =\Psi^{\prime}(A,[]) \\
\Psi^{\prime}(x M, l) & =x(\Psi M:: l) \\
\Psi^{\prime}((\lambda x . M) N, l) & =(\lambda x . \Psi M)(\Psi N:: l) \\
\Psi^{\prime}(A M, l) & =\Psi^{\prime}(A, \Psi M:: l)\end{aligned}$ & $\begin{aligned} \Theta(x[]) & =x \\
\Theta(x(u:: l)) & =\Theta^{\prime}(x \Theta u, l) \\
\Theta(\lambda x . t) & =\lambda x . \Theta t \\
\Theta((\lambda x . t)(u:: l)) & =\Theta^{\prime}((\lambda x . \Theta t) \Theta u, l) \\
& = \\
\Theta^{\prime}(A,[]) & =a p p(A) \\
\Theta^{\prime}(A, u:: l) & =\Theta^{\prime}(A \Theta u, l)\end{aligned}$ \\
\hline
\end{tabular}

Table 7. Translations $\Psi$ and $\Theta$

Proposition 6. $\Theta \circ \Psi=i d$ and $\Psi \circ \Theta=i d$.

Proof. Extend the proof in [2].

Lemma 3. $\Psi(M[N / x])=\operatorname{subst}(\Psi N, x, \Psi M)$.

Corollary 3. $\Theta(\operatorname{subst}(v, x, t))=\Theta t[\Theta v / x]$.

The promised isomorphism of normalisation procedures is the following

\section{Theorem 1.}

1. If $M \rightarrow_{\beta} M^{\prime}$ in $N J$ then $\Psi M \rightarrow_{\beta_{H}} \Psi M^{\prime}$ in $H J$.

2. If $t \rightarrow_{\beta_{H}} t^{\prime}$ in $H J$ then $\Theta t \rightarrow_{\beta} \Theta t^{\prime}$ in $N J$.

Hence $\beta$ and $\beta_{H}$ are isomorphic, but $\beta$ performs normalisation in $N J$ whereas $\beta_{H}$ performs cut elimination in $H J$. 


\section{Further Work}

There are two main directions of further work.

First, to extend this work to the other connectives of intuitionistic predicate logic. Challenging seems to be the positive fragment and the treatment in this framework of the anomalies caused by disjunction and reported in [15].

Second, to generalise the whole enterprise to classical logic. The key players should be Herbelin's $L K T$ and $\bar{\lambda}_{\mu}$-calculus [7], Parigot's natural deduction and $\lambda_{\mu}$-calculus [8] and an appropriate $L K^{t}$. We plan to report on this in [4].

\section{Acknowledgements}

We thank Samson Abramsky for encouragement and guidance, Luís Pinto for his enthusiasm about this work and René Vestergaard for many chats on structural proof theory.

\section{References}

1. V. Danos, J-B. Joinet, and H. Schellinx. A new deconstructive logic: linear logic. The Journal of Symbolic Logic, 62(2):755-807, 1997.

2. R. Dyckhoff and L. Pinto. Cut-elimination and a permutation-free sequent calculus for intuitionistic logic. Studia Logica, 60:107-118, 1998.

3. R. Dyckhoff and L. Pinto. Permutability of proofs in intuitionistic sequent calculi. Theoretical Computer Science, 212:141-155, 1999.

4. J. Espírito Santo, 2000. PhD Thesis (in preparation).

5. J. Gallier. Constructive logics. Part I. Theoretical Computer Science, 110:248-339, 1993.

6. H. Herbelin. A $\lambda$-calculus structure isomorphic to a Gentzen-style sequent calculus structure. In L. Pacholski and J. Tiuryn, editors, Proceedings of CSL'94, volume 933 of Lecture Notes in Computer Science, pages 61-75. Springer-Verlag, 1995.

7. H. Herbelin. Sequents qu'on calcule, 1995. PhD Thesis, Université Paris VII.

8. M. Parigot. $\lambda \mu$-calculus: an algorithmic interpretation of classic natural deduction. In Int. Conf. Logic Prog. Automated Reasoning, volume 624 of Lecture Notes in Computer Science. Springer Verlag, 1992.

9. G. Pottinger. Normalization as a homomorphic image of cut-elimination. Annals of Mathematical Logic, 12:323-357, 1977.

10. D. Prawitz. Natural Deduction. A Proof-Theoretical Study. Almquist and Wiksell, Stockholm, 1965.

11. A.M. Ungar. Normalization, Cut-eliminations and the Theory of Proofs. Number 28 in CSLI Lecture Notes. 1992.

12. C. Urban and G. Bierman. Strong normalisation of cut-elimination in classical logic. In Proceedings of TLCA'99, Lecture Notes in Computer Science. SpringerVerlag, 1999.

13. R. Vestergaard and J.Wells. Cut rules and explicit substitutions. In Second International Workshop on Explicit Substitutions, 1999.

14. P. Wadler. A Curry-Howard isomorphism for sequent calculus, 1993. Manuscript.

15. J. Zucker. The correspondence between cut-elimination and normalization. Annals of Mathematical Logic, 7:1-112, 1974. 